$\begin{gathered}\text { Revista do Departamento de Geografia } \\ \text { Universidade de São Paulo } \\ \text { www.revistas.usp.br/rdg }\end{gathered}$
Volume Especial - XVII SBGFA / I CNGF (2017)

\title{
Eventos Extremos de Precipitação no Perfil Longitudinal Paraty (RJ) - Campos do Jordão (SP)
}

\author{
Extreme Rainfall Events in the Longitudinal Profile \\ Paraty $(R J)$ - Campos do Jordão (SP)
}

\author{
Maria Rita Pelegrin de Oliveira \\ Universidade de São Paulo \\ maria.rita.oliveira@usp.br \\ Emerson Galvani \\ Universidade de São Paulo \\ egalvani@usp.br
}

\begin{abstract}
Recebido (Received): $\quad$ 18/03/2017
Aceito (Accepted): 10/06/2017
\end{abstract}

DOI: $10.11606 /$ rdg.v0ispe. 133419

Resumo: O objetivo deste trabalho foi analisar a variabilidade das precipitações e seus respectivos valores extremos ao longo do perfil longitudinal SE NW, entre os municípios de Paraty/RJ, Cunha/SP, Taubaté/SP e Campos do Jordão/SP. Para isso, foram utilizados dados secundários de séries temporais de 21 anos (entre 1982 - 2002) para cada localidade. Estas séries temporais apresentam valores dos acumulados diários de precipitação; com estes dados foram aplicadas técnicas estatísticas de análise de tendência central e box plot. Com o primeiro método, foram elaborados gráficos das médias mensais e dos volumes máximos de precipitação em 24 horas; já com o método box plot (quartis) foi obtida a distribuição estatística do volumes de precipitação e seus respectivos valores extremos, os também chamados de outliers. Como resultado, as análises apontaram para uma maior frequência de chuvas menos intensas ao longo do perfil longitudinal, porém com ocorrências de eventos extremos em $24 \mathrm{~h}$ que superaram em até $138,9 \%$ as médias mensais esperadas. A análise de tendência central mostrou resultados mais generalizdos, porém foi capaz de demonstrar as diferenças entre as máximas em $24 \mathrm{~h}$ e as médias mensais esperadas para cada localidade. Com a técnica de análise do box plot, os resultados foram mais detalhados, demonstrando de maneira mais nítida o variabilidade dos volumes de precipitação ao longo da série, incrementando, desta forma, a qualidade da análise da distribuição das precipitações.

Palavras chave: Análise Estatística; Quartil; Média Mensal; Outlier.

\begin{abstract}
The aim of this paper was to analyze the rainfall variability and their respective extreme values along the longitudinal profile SE - NW, among the municipalities of Paraty/RJ, Cunha/SP, Taubaté/SP and Campos do Jordão/SP. For that, was used secondary data of time series (between 1982 - 2002) for each locality. These time series presents values of accumulated daily rainfall; with these data, statistical techniques of central tendency analysis and box plot were applied. With the first method, were elaborated graphics of monthly averages and the maximum volumes of precipitation in $24-h$; with the box plot method (quartile method), the statistical distribution of precipitation volumes and their respective extreme values, called outliers, were obtained. As a result, the analyzes pointed to a higher frequency of less intense rainfall along the longitudinal profile, but with occurrences of extreme events in $24 \mathrm{~h}$ that exceeded by $138.9 \%$ the expected monthly averages. The central tendency analysis showed more generalized results, but was able to demonstrate the differences between the maximums in $24 \mathrm{~h}$ and the monthly averages expected for each locality. With the box plot analysis, the results were more detailed, demonstrating more clearly the variability of the precipitation volumes throughout the time series, thus increasing the quality of precipitation distribution analysis.
\end{abstract}

Keywords: Statistical Analysis; Quartile; Monthly Average; Outlier. 


\section{INTRODUÇÃO}

Os eventos naturais extremos de precipitação, segundo Monteiro e Zanella (2014), podem causar consequências graves especialmente em meio urbano. Estes eventos são os principais agentes deflagradores de deslizamentos de terra, fluxos de detritos, erosões, enchentes e inundações. O resultado da interação destes fenômenos com a população pode causar mortes, proliferação de doenças, desabrigados, feridos, danos à estrutura urbana e prejuízos econômicos. Ainda de acordo com os autores, as consequências dos eventos extremos são mais intensas em áreas habitadas por populações de baixa renda e em locais de urbanização precária - muitas vezes áreas frágeis do ponto de vista natural, tais como encostas íngremes e planícies de inundação fluvial.

Os eventos extremos de precipitação, de acordo com Andrade, Pinheiro e Neto (2015), são muito comuns na região Sudeste do Brasil ao longo da estação chuvosa. Os autores salientam que a particularidade de um evento extremo está no volume total acumulado de chuva em um determinado intervalo de tempo, por exemplo: o total de chuva acumulado em 24 horas. Segundo Amaral e Gutjahr (2011), o setor leste do estado de São Paulo, onde se localiza a área de estudo deste trabalho (Figura 01) - com exceção do município de Paraty, o qual está localizado no litoral sul do estado do Rio de Janeiro - está sujeito aos escorregamentos de encostas nas áreas do Planalto da Bocaina e nas escarpas da serra da Mantiqueira (onde estão respectivamente localizados os municípios de Cunha e Campos do Jordão); a Depressão do médio Paraíba do Sul, onde está localizado o município de Taubaté, está sujeita à inundações e enchentes. De acordo com as autoras, o litoral de São Paulo está sujeito à erosão costeira, ressacas e recalques de solo ao longo da orla; por similaridade física, podem-se aplicar estes tipos de ocorrências ao município de Paraty.

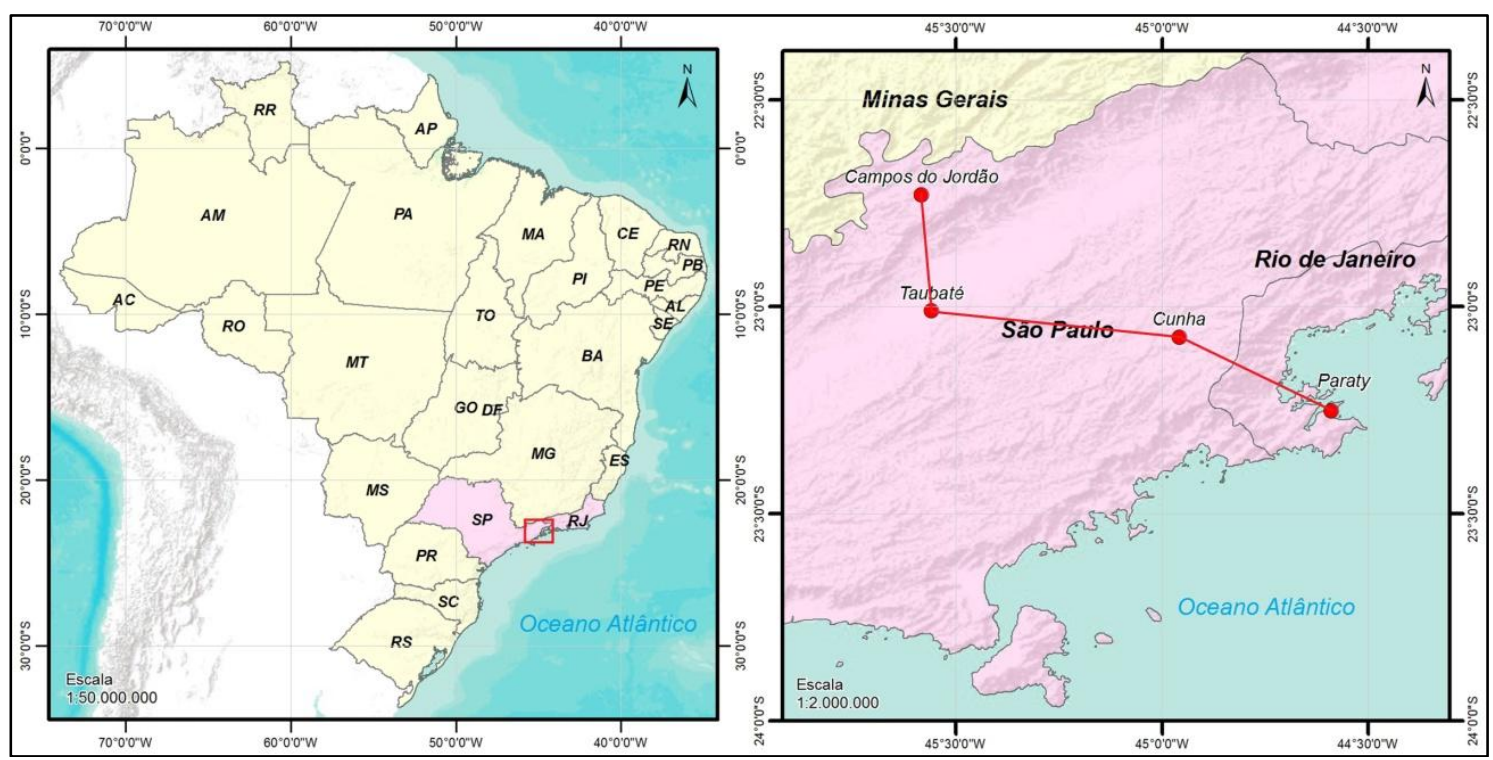

Figura 01: Localização da área de estudo.

Dada a importância do conhecimento do comportamento e das consequências dos eventos extremos em relação ao meio socioambiental, Monteiro e Zanella (2014) salientam que a utilização de meios estatísticos é fundamental para o entendimento destes fenômenos, podendo servir de ferramenta à gestão pública a fim de evitar e/ou mitigar os efeitos negativos que um evento extremo pode suscitar. Segundo Silvestre, San’tAnna Neto e Flores (2013), um problema muito comum em Climatologia é como classificar um conjunto de dados em classes e como definir valores habituais ou extremos. Para tentar responder estas questões, os autores resgataram e testaram diversos métodos estatísticos a fim de definir anos-padrão para os dados de temperatura e precipitação da Estação Meteorológica da FCT/UNESP do município de Presidente Prudente/SP. Dentre os métodos utilizados, o Box Plot foi testado e, segundo os autores, a vantagem do método consiste em fornecer uma visualização gráfica e rápida da distribuição dos dados, além de informar sobre a simetria da variabilidade das precipitações: "se a distribuição é simétrica, a caixa encontra-se equilibrada com a mediana, se posicionando no centro da mesma; já para distribuições assimétricas, há um desequilíbrio na caixa com relação à mediana" (SILVESTRE et al., 2013, p.27). 
Para Schneider e Silva (2014), a utilização de métodos estatísticos na análise é de grande valia, pois estes métodos auxiliam em uma abordagem mais apropriada no estudo da variabilidade dos atributos do clima. Os autores aplicaram o método do Box Plot na identificação de anos-padrão secos e chuvosos para a microrregião de Dourados/MS. Para os autores, o método do Box Plot permitiu uma eficiente classificação dos anos-padrão em cinco categorias: habitual, seco, chuvoso, super seco e super chuvoso. Além disso, os autores apontam uma relevância do método para a Climatologia Geográfica, pois o Box Plot permitiu analisar os resultados sob o ponto de vista temporal, quantitativo e qualitativo.

Galvani e Luchiari (2005) aplicaram o método do Box Plot com o objetivo de determinar os limites numéricos de regimes pluviométricos considerados como normal, úmido, super úmido, seco e super seco para os dados da estação meteorológica da ESALQ/USP em Piracicaba/SP. Segundo os autores, a aplicação do método do Box Plot permitiu classificar os regimes pluviométricos de maneira rápida e objetiva, principalmente quando se trabalha com longas séries históricas. Além destas características, o Box Plot também informa os valores outliers (valores extremos de uma série), o qual foi útil na determinação dos meses super secos e super úmidos. Desta forma, o objetivo deste artigo é investigar o comportamento dos eventos de precipitação extrema ao longo do transecto Paraty/RJ - Campos do Jordão/SP a partir da análise estatística de tendência central e por meio da técnica do Box Plot, a qual é capaz de informar valores mínimos, medianas, valores máximos e extremos de uma dada série de dados, assim como seus outliers.

\section{MATERIAIS E MÉTODOS}

Para a realização deste trabalho, foram coletados dados secundários de precipitação acumulada em 24h, expressos em mm, de quatro postos pluviométricos referentes aos municípios da área de estudo (Tabela 01), estes dados foram obtidos por meio das publicações do sítio eletrônico da Agência Nacional de Águas/Hidroweb. Este trabalho utilizou uma série temporal de 21 anos de observação, com início em 1982 e término no ano de 2002. Chegou-se a este intervalo de tempo em função deste apresentar o período de melhor qualidade dos dados, objetivando diminuição do preenchimento de falhas dos bancos de dados. Mesmo assim, houve a necessidade do preenchimento dos dados faltantes, os quais ocorreram nas séries dos postos de Paraty-RJ e Campos do Jordão-SP. Para efetuar o preenchimento dos dados faltantes, as lacunas foram completadas por meio da média aritmética para cada dia incompleto.

Tabela 01: Localização dos postos pluviométricos e suas respectivas falhas em banco de dados

\begin{tabular}{|c|c|c|c|c|c|c|c|}
\hline \multicolumn{7}{|c|}{ Postos de Observação - Agência Nacional de Águas (ANA) } \\
\hline Município & Estado & Postos & X (m) & Y (m) & Altitude (m) & Falha (\%) & Operadora \\
\hline Paraty & RJ & Parati & 524157 & 7431697 & 19 & 3,25 & CPRM \\
\hline Cunha & SP & $\begin{array}{c}\text { Alto S. do } \\
\text { Mar }\end{array}$ & 514444 & 7439459 & 1270 & 0,00 & CPRM \\
\hline Taubaté & SP & Taubaté & 442740 & 7451995 & 614 & 0,00 & CPRM \\
\hline $\begin{array}{c}\text { Campos } \\
\text { do Jordão }\end{array}$ & SP & $\begin{array}{c}\text { Campos do } \\
\text { Jordão }\end{array}$ & 450361 & 7489600 & 1620 & 0,11 & DAEE \\
\hline
\end{tabular}

Os dados de precipitação foram trabalhados por meio de duas abordagens estatísticas. A primeira consistiu no cálculo das medidas de tendência central, na totalização das médias mensais e dos valores máximos em 24h para cada posto pluviométrico ao longo da série de 21 anos. Os resultados foram expressos por meio de gráficos de barras confeccionados no software Microsoft Excel. A segunda abordagem estatística consistiu em elaborar gráficos do tipo Box Plot (gráfico de box). Este tipo de gráfico permite a obtenção e a representação dos valores mínimos, medianas e os valores máximos por meio de um retângulo constituído com os quartis Q1 (o qual representa 25\% da distribuição dos dados série), Q2 ou mediana (o qual representa $50 \%$ da distribuição dos dados série) e Q3 (o qual representa $75 \%$ da distribuição dos dados série), além dos valores outliers (Figura 02). Para isso, foram considerados somente os valores maiores ou iguais a $0,1 \mathrm{~mm}$ de chuva, descartando os valores nulos das séries. Após isto, os dados de precipitação foram ordenados de forma crescente (do menor para o maior) e calculadas a moda e a média dos dias de ocorrência 
de chuva. Para a obtenção dos gráficos Box Plot e seus respectivos valores de Q1, Q2, Q3, valor máximo, mínimo e outlier, foi utilizado o software Origin 6.0.

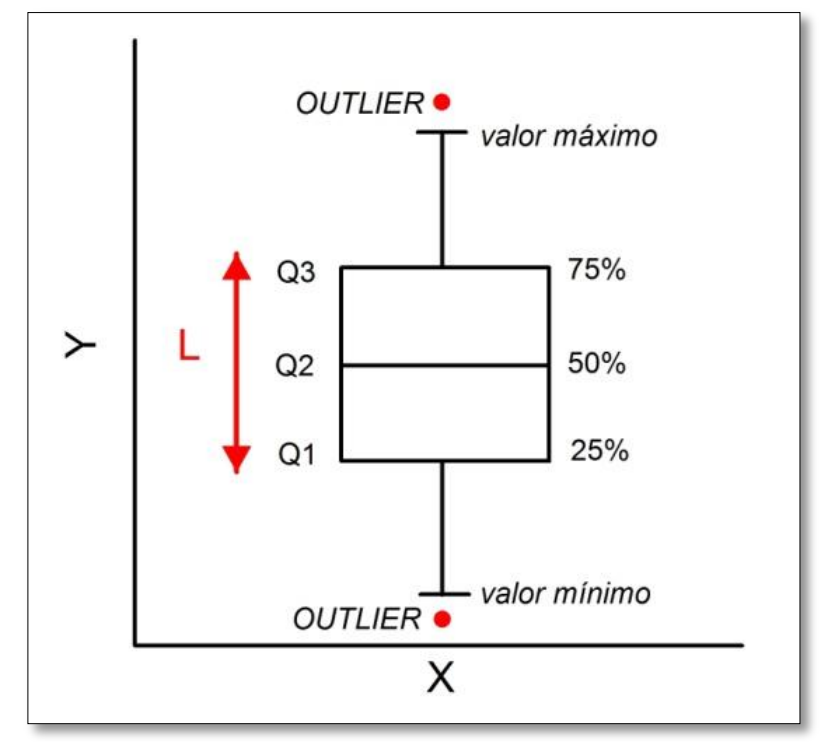

Figura 02: Elementos constituintes do Box Plot: Q1, Q2 (mediana), Q3, valor mínimo, valor máximo e outlier.

Segundo Figueira (1998), os valores máximos e os outliers são valores que apresentam um distanciamento em relação aos demais valores de um determinado conjunto de dados; são designados como "anormais", "contaminantes" ou "extremos". Em alguns casos, estes valores discrepantes podem apontar para uma possível inconsistência em um conjunto de dados, porém, no caso deste trabalho, são estes valores discrepantes que interessam. Pois é por meio dos valores máximos e dos outliers que se podem identificar os valores extremos um conjunto de dados de uma maneira não subjetiva. A partir dos valores fornecidos pelo Box Plot, podem-se calcular os pontos chamados de "extremos". Segundo Figueira (1998), para obtermos os outliers e os valores extremos de um conjunto de dados, devem-se ser aplicadas as seguintes equações: Q3 + 1,5L e Q3 + 3L. Os valores que estiverem no intervalo dado por estas equações podem ser classificados como outliers; os valores resultantes maiores que Q3 + 3L são considerados como extremos.

\section{RESULTADOS E DISCUSSÕES}

A partir de uma visão panorâmica, a série temporal de 21 anos (1982-2002) para todos os postos pluviométricos da área de estudo, independentemente de suas localizações a barlavento e/ou a sotavento ao longo do transecto, apresentam médias mensais que variam de acordo com as características do clima tropical, onde há um maior maior aporte de chuvas nos meses de primavera e verão, e uma diminuição dos totais de precipitação nos meses de outono e inverno. Porém, analisando em um maior detalhe, nota-se o efeito orográfico nos totais de chuva postos de Cunha/SP e Campos do Jordão/SP, onde estão concentrados os maiores volumes de precipitação (Figuras 03, 04, 05 e 06).

Do ponto de vista da relação entre as médias mensais calculadas para os postos pluviométricos da área de estudo e seus respectivos máximos acumulados em $24 \mathrm{~h}$, pode-se afirmar que as quatro localidades apresentaram meses em que os máximos em $24 \mathrm{~h}$ superaram as médias mensais esperadas. Segundo Galvani (2005), os valores máximos podem apresentar uma maior importância do que as médias, pois estas são valores relativos e não necessariamente representam a realidade da variabilidade de um determinado atributo do clima.

O posto de Paraty/RJ (Figura 03) foi o que apresentou uma maior quantidade de ocorrências de valores máximos em $24 \mathrm{~h}$ que superaram a média mensal esperada. Respectivamente, para o mês de fevereiro, houve um incremento de 16,6\%; para o mês de abril, houve um incremento de 27,2\%; para o mês de junho, precipitou $116,5 \%$ a mais que a média esperada para mês; para o mês de julho, houve um aumento de 138,9\%; para o mês de agosto, precipitou $12,1 \%$ a mais que a média; e, por fim, para o mês de outubro, houve um aumento de $63,1 \%$ das chuvas esperadas para o mês. 
O posto de Cunha/SP (Figura 04) foi o que apresentou a menor quantidade de ocorrências de valores máximos em $24 \mathrm{~h}$ que superaram a média mensal esperada, duas no total. No mês de julho houve um acréscimo de $2 \%$ e no mês de agosto houve um aumento de $97,3 \%$ das chuvas esperadas para o mês. O posto de Taubaté/SP (Figura 05) apresentou quatro ocorrências de valores máximos em $24 \mathrm{~h}$ superiores às médias mensais - ocorreram nos meses de abril, junho, julho e agosto. Respectivamente, os acréscimos foram de: $6 \%, 69 \%, 46,9 \%$ e 22,6\%. Já o posto de Campos do Jordão/SP (Figura 06) apresentou três ocorrências onde os valores máximos em $24 \mathrm{~h}$ superaram as médias - ocorreram nos meses de junho, julho e agosto. Respectivamente, os acréscimos foram de: 44,7\%, 135,5\% e 7\%. Com exceção do posto de Paraty/RJ, os demais postos concentraram as ocorrências de valores máximos em $24 \mathrm{~h}$ superiores às médias mensais nos meses de outono e inverno.

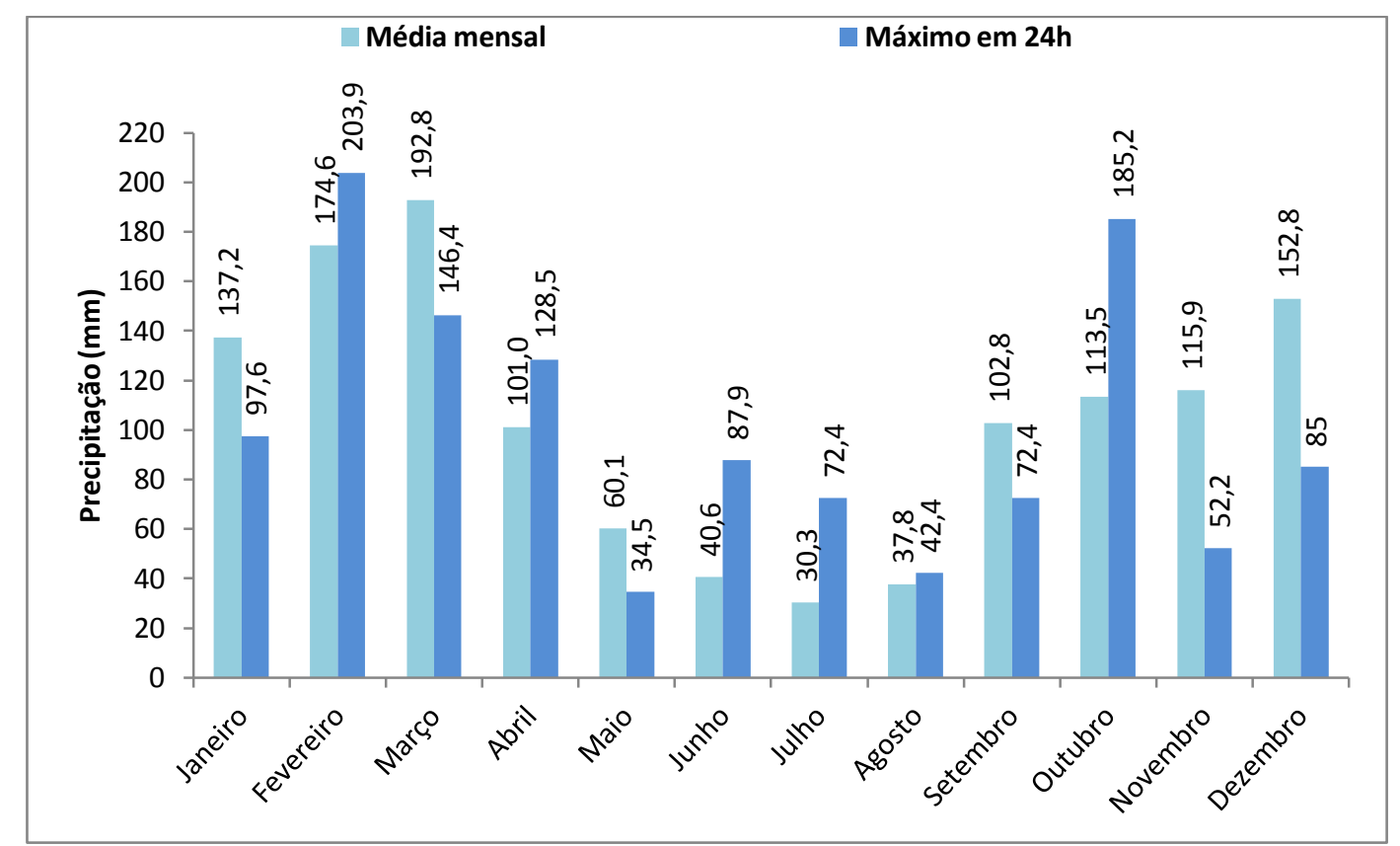

Figura 03: Média mensal versus precipitação máxima em 24h para Paraty-RJ (1982-2002).

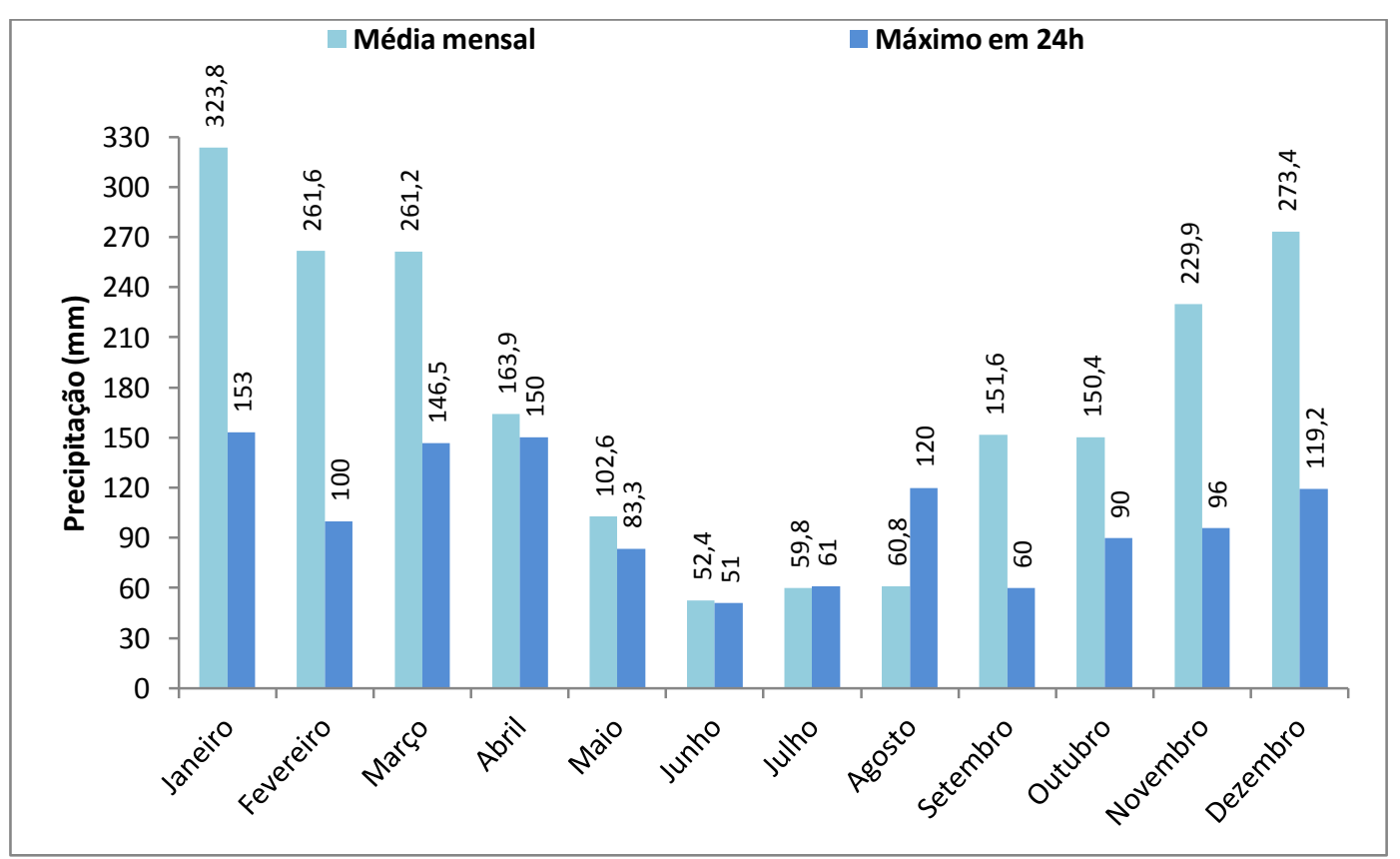

Figura 04: Média mensal versus máximo de precipitação em 24h para Cunha-SP (1982-2002). 


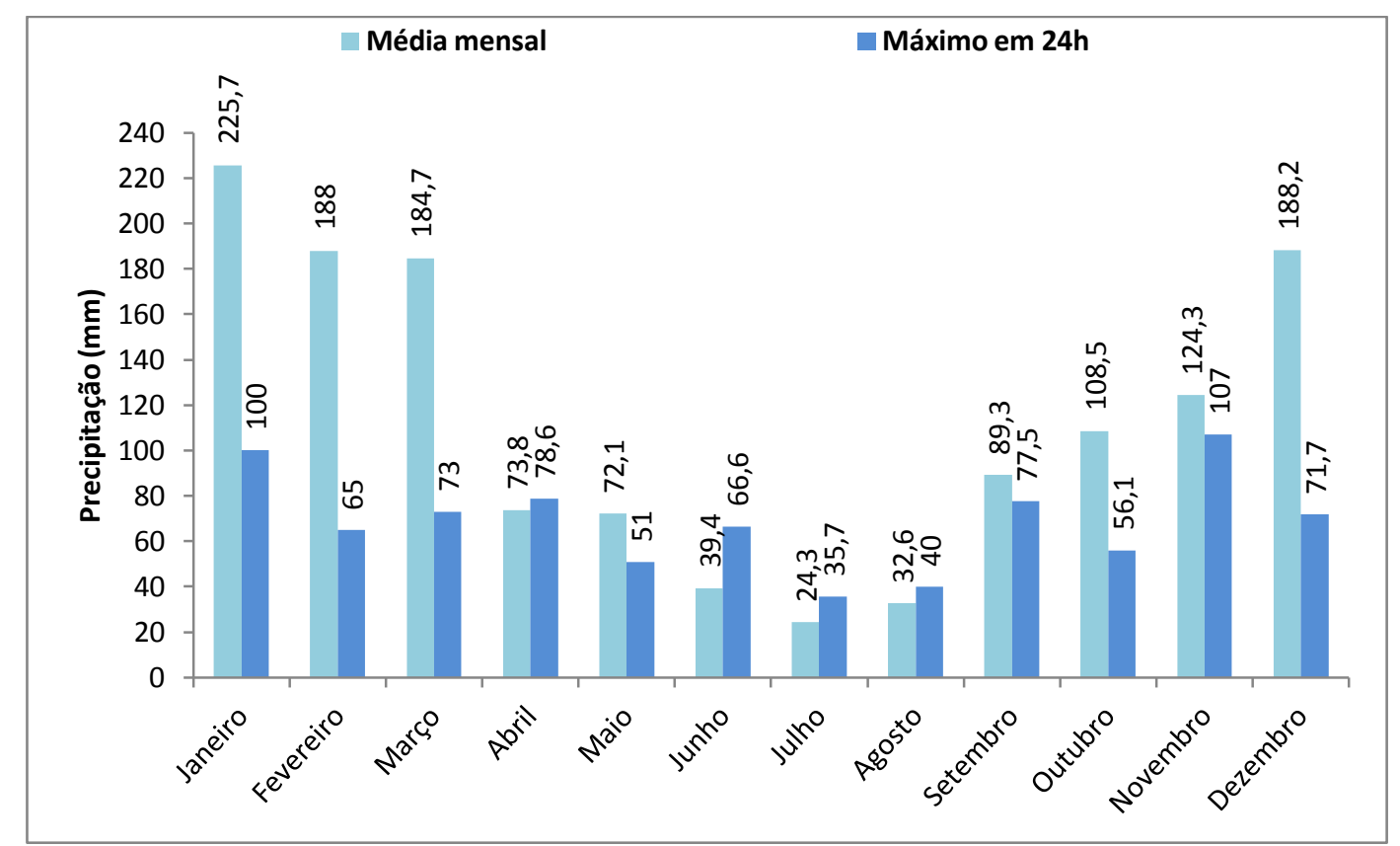

Figura 05: Média mensal versus precipitação máxima em 24h para Taubaté-SP (1982-2002).

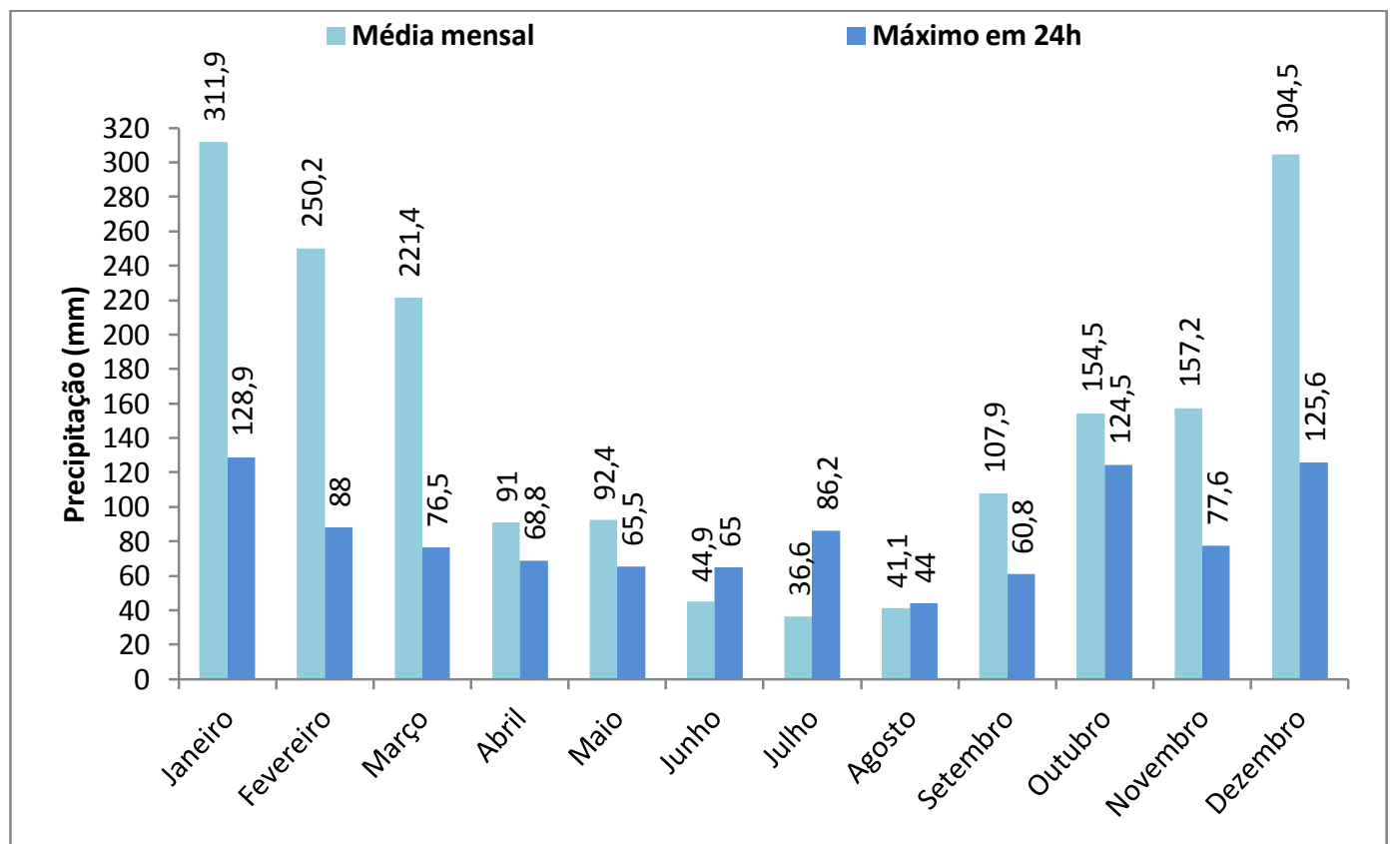

Figura 06: Média mensal versus máximo de precipitação em 24h para Campos do Jordão-SP (1982-2002).

A segunda etapa da análise estatística dos dados de precipitação da área de estudo consistiu na investigação dos quartis (Q1, Q2 e Q3), valores mínimos, valores máximos e outliers dos dias de volumes de chuva maiores ou igual 0,1 mm; onde, de 7670 dias totais (de 1982-2002), o posto de Paraty/RJ apresentou 3014 dias de chuva, o posto de Cunha/SP apresentou o total de 3584, o posto de Taubaté/SP apresentou 2083 ocorrências e o posto de Campos do Jordão exibiu 3439 dias com ocorrências de chuva. O Box Plot do posto de Paraty/RJ (Figura 07), mostrou que o primeiro quartil (Q1) da série apresenta valores iguais ou menores que $1,8 \mathrm{~mm}$; o segundo quartil (Q2) compreende valores iguais ou inferiores a 4,2mm e o terceiro quartil (Q3) apresenta valores iguais ou menores que $9,8 \mathrm{~mm}$; valor máximo de $58,3 \mathrm{~mm}$ e outlier de $203,9 \mathrm{~mm}$. O Box Plot para o posto de Cunha/SP (Figura 08) apresentou valores de primeiro quartil (Q1) iguais ou inferiores a $1,9 \mathrm{~mm}$; o segundo quartil (Q2) valores iguais ou inferiores a $6,9 \mathrm{~mm}$ e o terceiro quartil (Q3) valores iguais ou inferiores a $16,9 \mathrm{~mm}$; valor máximo de $7 \mathrm{~mm}$ e outlier de $153 \mathrm{~mm}$. Para o posto de Taubaté/SP (Figura 09), o Box Plot apontou para valores do primeiro quartil (Q1) iguais ou inferiores a $4 \mathrm{~mm}$; segundo quartil (Q2) valores iguais ou inferiores a $9 \mathrm{~mm}$ e terceiro quartil (Q3) valores iguais ou inferiores a $18,6 \mathrm{~mm}$; valor máximo de $64,2 \mathrm{~mm}$ e outlier de $107 \mathrm{~mm}$. 
O Box Plot referente ao posto de Campos do Jordão/SP (Figura 10) exibiu valores de primeiro quartil (Q1) iguais ou inferiores a $1,7 \mathrm{~mm}$; segundo quartil $(\mathrm{Q} 2)$ valores iguais ou inferiores a $6,1 \mathrm{~mm}$ e valores de terceiro quartil (Q3) iguais ou inferiores a $14,8 \mathrm{~mm}$; valor máximo de $67,5 \mathrm{~mm}$ e outlier de $128,9 \mathrm{~mm}$. Analisando os valores de moda e dos quartis para os postos da área de estudo, nota-se valores de baixa intensidade de precipitação. O posto de Paraty/RJ apresenta o valor de $2,4 \mathrm{~mm}$ como o de maior frequência para a série; o posto de Cunha/SP apresenta o valor de $0,1 \mathrm{~mm}$; para o posto de Taubaté/SP, a moda é de $5 \mathrm{~mm}$ e para o posto de Campos do Jordão/SP o valor é de $0,3 \mathrm{~mm}$. Os valores da moda para os postos de Cunha/SP e Campos do Jordão/SP, os quais se localizam a barlavento e em uma altitude de $1270 \mathrm{~m}$ e $1620 \mathrm{~m}$ respectivamente, podem sugerir que a formação de nevoeiros orográficos e formação de orvalho são frequentes nestas localidades.

Segundo Figueira (1998), para determinar valores extremos de uma série de dados segundo o método do Box Plot, deve-se aplicar a equação Q3 + 3L. Os valores acima do resultado desta equação são considerados extremos. Calculados os valores extremos para os postos de Paraty/RJ, Cunha/SP, Taubaté/SP e Campos do Jordão/SP, temos os respectivos resultados: $33,8 \mathrm{~mm}, 61,9 \mathrm{~mm}, 62,4 \mathrm{~mm}$ e $54,1 \mathrm{~mm}$. Comparando os valores extremos com os respectivos outliers de cada posto, para Paraty/RJ há uma diferença de 503,2 \%; para Cunha/SP a diferença é de 147,1\%; para Taubaté/SP, há uma diferença de 71,4\% e para o posto de Campos do Jordão/SP, a diferença é de 138,2\% (Tabela 02). Este tipo de informação mostra que, mesmo em séries que predominam chuvas de baixa intensidade, não pode-se descartar os eventos extremos, os outliers das séries em especial.

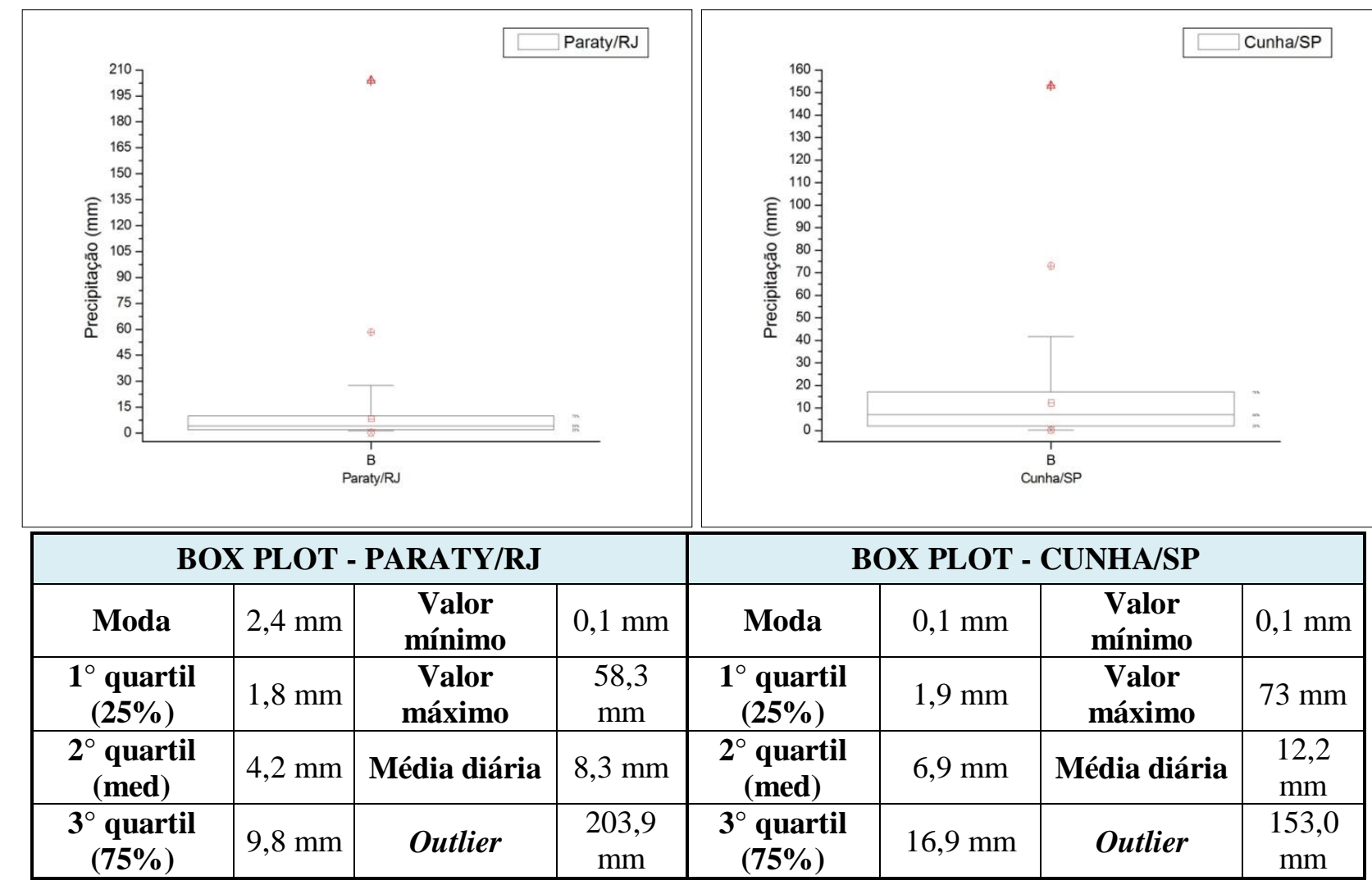

Figura 07: Box Plot para o posto de Paraty/RJ. Figura 08: Box Plot para o posto de Cunha/SP 


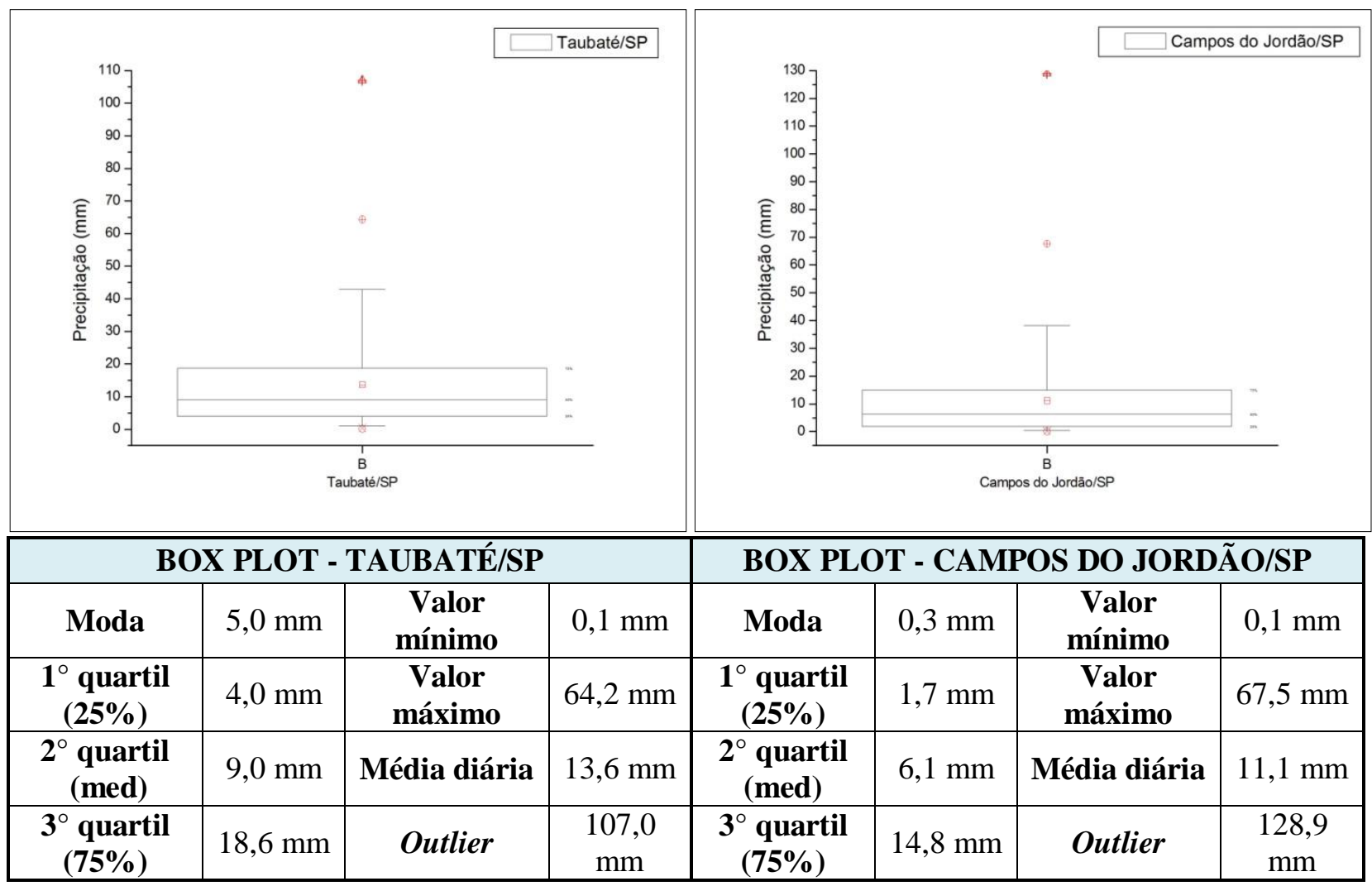

Figura 09: Box Plot para o posto de Taubaté/SP. Figura 10: Box Plot para o posto de Campos do Jordão/SP

Tabela 02: Valores extremos, outliers e diferença em porcentagem.

\begin{tabular}{|c|c|c|c|}
\hline \multicolumn{4}{|c|}{ VALORES EXTREMOS E OUTLIERS } \\
\hline POSTOS & Q3 + 3L & OUTLIERS & ACRÉSCIMO (\%) \\
\hline PARATY/RJ & $33,8 \mathrm{~mm}$ & $203,9 \mathrm{~mm}$ & 503,2 \\
\hline CUNHA/SP & $61,9 \mathrm{~mm}$ & $153 \mathrm{~mm}$ & 147,1 \\
\hline TAUBATÉ/SP & $62,4 \mathrm{~mm}$ & $107 \mathrm{~mm}$ & 71,4 \\
\hline CAMPOS DO JORDÃO/SP & $54,1 \mathrm{~mm}$ & $128,9 \mathrm{~mm}$ & 138,2 \\
\hline
\end{tabular}

Do ponto de vista dos outliers, observa-se que a intensificação orográfica se fez presente nos municípios de Cunha/SP e Campos do Jordão/SP, localizados a barlavento ao longo do transecto Paraty/RJ Campos do Jordão/SP, exibindo os valores de $153 \mathrm{~mm}$ e $128,9 \mathrm{~mm}$ respectivamente. O valor outlier para o posto de Paraty/RJ mereceria um estudo sinótico detalhado para a compreensão da origem do valor extremo de $203,9 \mathrm{~mm}$ de chuva em $24 \mathrm{~h}$.

\section{CONSIDERAÇÕES FINAIS}

Após calculadas as medidas de tendência central e os valores dos quartis (Q1, Q2 e Q3), valor máximo, valor extremo e outliers, notou-se que apesar das séries dos postos da área de estudo apresentarem uma maior frequência de chuvas menos intensas, os eventos extremos devem ser considerados devido à grande intensidade destes. Por uma análise de tendência central, destaca-se aqui o posto de Paraty/RJ, o qual apresentrou seis meses de precipitações acima da média mensal esperada. Este resultado obtido por meio desta metodologia já seria interessante no âmbito da gestão pública. Quando se aplica o método do Box Plot, os resultados são mais detalhados, demonstrando de maneira mais nítida o variabilidade dos dados ao longo da série, incrementando, desta forma, a qualidade da análise do variabilidade das precipitações. 


\section{BIBLIOGRAFIA}

AMARAL, Rosangela do; GUTJAHR, Miriam Ramos. Desastres naturais (Série Cadernos de Educação Ambiental, 8). São Paulo: IG/SMA, 2011.

ANDRADE, Kelen Martins; PINHEIRO, Henri Rossi; NETO, Giovanni Dolif. Evento extremo de chuva no Rio de Janeiro: análise sinótica, previsão numérica e comparação com eventos anteriores. Ciência e Natura, Santa Maria, v.37, Ed. Especial SIC, 2015, p.175 - 180. Revista do Centro de Ciências Naturais e Exatas - UFSM.

FIGUEIRA, Maria Manuela Caria. Identificação de Outliers. MILLENIUM nº12 - Outubro de 1998. Disponível em <http://www.ipv.pt/millenium/arq12.htm>. Acesso em março de 2017.

GALVANI, Emerson. Sistematização de Dados Quantitativos. In: Praticando geografia: técnicas de campo e laboratório. ORG: VENTURI, Luis Antonio Bittar - São Paulo: Oficina de Textos, 2005 - p.175 -186 .

GALVANI, Emerson; LUCHIARI, Ailton. Critérios para classificação de anos com regime pluviométrico normal, seco e úmido ${ }^{1}$. Anais do X Encontro de Geógrafos da América Latina - 20 a 26 de março de 2005 - Universidade de São Paulo - p. 5701 - 5710.

MONTEIRO, Jander Barbosa; ZANELLA, Maria Elisa. Eventos pluviométricos extremos e impactos associados em Fortaleza/Brasil: uma análise a partir da técnica dos quantis. In: Experimentos em Climatologia Geográfica. ORG: SILVA, CHARLEI Aparecido; FIALHO, Edson Soares e STEINKE, Ercília Torres - Dourados, MS: UFGD, 2014 - p. 165 - 184.

SCHNEIDER, Heverton; SILVA, Charlei Aparecido da. O uso do modelo box plot na identificação de anospadrão secos, chuvosos e habituais na microrregião de Dourados, Mato Grosso do Sul. Revista do Departamento de Geografia - USP, Volume 27 (2014), p. 131-146.

SILVESTRE, Miriam Rodrigues; SANT’ANNA NETO, João Lima; FLORES, Edilson Ferreira. Critérios estatísticos para definir anos padrão: uma contribuição à climatologia geográfica. Revista Formação, n.20, volume $2(2013)-$ p. $23-53$. 